IOS Press

\title{
Editorial
}

\section{The Semantic Web for all}

\author{
Christophe Guéret ${ }^{\mathrm{a}}$, Stephane Boyera ${ }^{\mathrm{b}}$, Mike Powell ${ }^{\mathrm{c}}$ and Martin Murillo ${ }^{\mathrm{d}}$ \\ ${ }^{a}$ Data Archiving and Networked Services (DANS), Anna van Saksenlaan 51, 2593 HW Den Haag, The Netherlands \\ E-mail: christophe.gueret@dans.knaw.nl \\ ${ }^{\mathrm{b}}$ SB Consulting (SBC4D), 5 rue de la Champagne 31100 Toulouse, France \\ E-mail: stephane@sbc4d.com \\ ${ }^{\mathrm{c}}$ IKM Emergent Programme, Kaiser Friedrich Straße 11, D-53113 Bonn, Germany \\ E-mail: mike@ikmemergent.net \\ ${ }^{\mathrm{d}}$ Notre Dame Global Adaptation Index (ND-GAIN), 1400 E. Angela Blvd., Suite 117, South Bend, IN 46617, USA \\ E-mail: murillo@ieee.org
}

\begin{abstract}
Over the past few years Semantic Web technologies have brought significant changes in the way structured data is published, shared and consumed on the Web. Emerging online applications based on the Web of Objects or Linked Open Data can use the Web as a platform to exchange and reason over semantically rich data covering any topic. Yet two-thirds of the world population is deprived of Web access and is thus deprived from applications that could have a critical impact on their life. There is a need to adapt Semantic Web technologies to put them to work "for all" in challenging contexts. This special issue describes progress made towards reaching that goal.
\end{abstract}

Keywords: ICT4D, Semantic Web, knowledge representation, ontology, crisis response

\section{Editorial}

Over the past few years Semantic Web technologies have brought significant changes in the way structured data is published, shared and consumed on the Web. Emerging online applications based on the Web of Objects or Linked Open Data can use the Web as a platform to exchange and reason over semantically rich data covering any topic. This jump into what is often referred to as the "Web 3.0" holds the promises of an increased support from computers which will be able to understand and reason over our world with outcomes ranging from more timely, accurate and efficient information to computer supported scientific discoveries. The latent pitfall of this technical and societal advances is that these emerging technologies typically assume that connectivity to data repositories and entity resolution services are always available. This may not be a valid assumption in many cases. Indeed, there are about 4.5 billion people in the world who have no or limited Internet access. Many data-driven applications may have a critical impact on the life of those people, but are inaccessible to those populations due to the architecture of today's Web based data platforms and the computing resources required to process them.

One example of this specific context is disaster response. Because of the chaotic nature of the environment most of the data exchange in this field still happens mostly manually but the emergency of the situation calls for more optimised data flows. In their paper entitled "The Humanitarian eXchange Language: Coordinating Disaster Response with Semantic Web Technologies", Carsten Keßler and Chad Hendrix highlight how Semantic Web technologies can be leveraged to improve information flow in the humanitarian domain and help decision makers better allocate resources and activities. A formal ontology is proposed 
as well as a matching infrastructure taking into account the commonly observed lack of Internet connectivity.

Being able to better express and share data is not the only hurdle to overcome. When considering target users that have limited education and/or can only speak in their own dialect literacy and semantics become an issue. The design of a voice-based interface between a user speaking his own language and a knowledge base containing multilingual factual information is a challenge Victor de Boer, Nana Baah Gyan, Anna Bon, Wendelien Tuyp, Chris van Aart and Hans Akkermans took up. Their paper "A Dialogue with Linked Data: Voice-based Access to Market Data in the Sahel" contains a description of a system that let users enter and query for market data by talking to their basic mobile phones. The bridge they propose between Linked Open Data and Voice is a step towards a more inclusive Web of Data that goes beyond text centric, and often English focused, interfaces.

As hinted earlier reasoning can play an important role in helping individuals making more out of data. Centralized computing is still the most common paradigm for this data processing where all the data is gathered into one system having enough computing power to make all the deductions. This paradigm is however challenged by hostile environments where such a large system is not available or when the centralization of data is not possible for privacy or technical reasons. Wei Tai, John Keeney and Declan O'Sullivan propose to push the intelligence back to the device layer to reason closer to the data. They describe in their paper "Resource-Constrained Reasoning Using a Reasoner Composition Approach" how a composition approach to reasoning can be taken to fit the different resources and datasets at hand. By selecting rules to be applied to a dataset a more resourceefficient amount of reasoning can be performed.

The three papers of this special issue are all steps towards "The Semantic Web for all". They highlight the concrete role Semantic Web technologies can play in constrained scenarios and what is needed to adapt main-stream approaches to fit these specific contexts. It is however too soon to claim victory and move onto something else. The many specific challenges of these specific contexts (limited infrastructure, different societal situations, high illiteracy, multilinguality, ... ) call for having more Semantic Web researchers integrate these into their own respective research. This will be the key to drive Semantic Web research into producing more inclusive output that will be then more likely to be usable and used world wide. This shared objective is what brings together the growing "World Wide Semantic Web" community ${ }^{1}$ of Semantic Web researchers, humanitarian, development experts and social scientists.

${ }^{1}$ See http://worldwidesemanticweb.org/. 